\title{
Conserved Vectors for a Model of Nonlinear Atmospheric Flows Around The Rotating Spherical Surface
}

\author{
A.M. Araslanov ${ }^{1}$, L.R. Galiakberova ${ }^{1}$, N.H. Ibragimov ${ }^{1}$, R. N. Ibragimov $^{2 *}$ \\ ${ }^{1}$ Laboratory "Group analysis of mathematical models in natural and engineering sciences" \\ Ufa State Aviation Technical University 12, K. Marx, Str., 450000 Ufa, Russia \\ 2 Department of Mathematics University of Texas at Brownsville Brownsville, TX 78520, USA
}

\begin{abstract}
We derive the conserved vectors for the nonlinear two-dimensional Euler equations describing nonviscous incompressible fluid flows on a three-dimensional rotating spherical surface superimposed by a particular stationary latitude dependent flow. Under the assumption of no friction and a distribution of temperature dependent only upon latitude, the equations in question can be used to model zonal west-to-east flows in the upper atmosphere between the Ferrel and Polar cells. As a particualr example, the conserved densities are analyzed by visualizing the exact invariant solutions associated with the given model for the particular form of finite disturbances for which the invariant solutions are also exact solutions of Navier-Stokes equations.
\end{abstract}

Keywords and phrases: nonlinear Navier-Stokes equations, atmospheric waves, exact solutuons, nonlinear conservation laws

Mathematics Subject Classification: 35A20

\section{Introduction}

The presence of Coriolis effects originates the general west-to-east flows caused by the Earth's rotation which are related to jet streams, i.e., zones of fastmoving west-to east winds in the upper atmosphere between the Ferrell and Polar cells. Such flows exist due to the presence of the cold North and South poles and warm equator so that the pressure is low at high latitudes above the poles and is high in the temperature zones and even higher in the equatorial zones.

In almost all oceanographic applications, e.g., meteorology, the effects of rotation and the spherical shape of the Earth cannot be ignored and so the large-scale atmospheric dynamics is usually described by latitude-dependent Euler or Navier-Stokes three- and two-dimensional flows on a rotating spherical surface ([6],[7]; [25], [26], [20], [18]) or within the theory of shallow water approximation (see e.g., [36], [31], [23]; [21] and [35]). In particular, since non-steady westerly winds in the southern hemisphere are an important force contributing to climate modulation $([34],[1])$, understanding of the large-scale atmospheric dynamics is the topic of current extensive research. For example, recent studies by Shindell and Schmidt,[28] suggest

\footnotetext{
${ }^{*}$ Corresponding author. E-mail: Ranis. Ibragimov@utb.edu
} 
that global worming has increased the temperature difference between the southern, mid-, and highlatitudes and loss of Antarctic ozone have contributed to this increase. Additionally, as has been shown in [11], owing to the Coriolis forces, the temperature difference between the equator and the poles of a sphere gives rise to the waves advancing in the direction of the meridian and the waves propagating along the circles of latitude. The atmospheric motion modulated by the pressure distribution resulting from the combination of the latter two groups of intersecting waves give rise to the cyclonic and anticyclonic phenomena which are nowadays a paramount topic of research in atmospheric modeling. Particularly, the mechanisms of cyclone formation in the Earth's atmosphere have been recently studied by Belotserkovskii et al. [4] by means of numerical modeling of the complete gas-dynamic equations. Recent laboratory experiments on cyclone and anticyclone formation in a rotating stratified fluid have been studied in [8]. The importance of understanding the formation of cyclones and their time evolution in the Earth's atmosphere for the creation and distribution of weather systems throughout the world is summarized in Summerhayes 83 Thorpe [30].

The problem forming our main focus of interest here is to consider long waves progressing in the earth's atmosphere, which are captured by the rotation of the earth with the further goal to analyze and visualize the effects of rotation on the conserved densities corresponding to the Lagrangian associated with the nonlinear Euler equations modeling nonlinear atmospheric motion around the rotating earth. The possibility of extension to the case of Navier-Stokes flows is also discussed in this work.

The inquiry is motivated by dynamically significant Coriolis forces in modeling of large-scale oceanic and atmospheric motion with applications to meteorology, climate variability models, the general atmospheric circulation model, weather prediction, and a variety of applications to large-scale dispersant operations e.g. Deepwater Horizon incident studied recently in[12].

For completeness, we write explicitly the three-dimensional Navier-Stokes (NS) equations in spherical coordinates using the notation as in Bachelor, [2] (see also Lamb, [24]):

$$
\begin{gathered}
\frac{\partial u_{r}}{\partial t}+u_{r} \frac{\partial u_{r}}{\partial r}+\frac{u_{\theta}}{r} \frac{\partial u_{r}}{\partial \theta}+\frac{u_{\phi}}{r \sin \theta} \frac{\partial u_{r}}{\partial \phi}-\frac{u_{\theta}^{2}+u_{\phi}^{2}}{r} \\
=-\frac{\partial p}{\partial r}+\nu\left(\Delta u_{r}+\frac{2}{r} \frac{\partial u_{r}}{\partial r}+\frac{2 u_{r}}{r^{2}}\right) \\
\frac{\partial u_{\theta}}{\partial t}+u_{r} \frac{\partial u_{\theta}}{\partial r}+\frac{u_{\theta}}{r} \frac{\partial u_{\theta}}{\partial \theta}+\frac{u_{\phi}}{r \sin \theta} \frac{\partial u_{\theta}}{\partial \phi}+\frac{u_{r} u_{\theta}}{r}-\frac{u_{\phi}^{2} \cot \theta}{r}-2 \Omega \cos \theta u_{\phi} \\
=-\frac{1}{r} \frac{\partial p}{\partial \theta}+\nu\left(\Delta u_{\theta}+\frac{2}{r^{2}} \frac{\partial u_{r}}{\partial \theta}-\frac{u_{\theta}}{r^{2} \sin ^{2} \theta}-\frac{2 \cos \theta}{r^{2} \sin ^{2} \theta} \frac{\partial u_{\phi}}{\partial \phi}\right) \\
\frac{\partial u_{\phi}}{\partial t}+u_{r} \frac{\partial u_{\phi}}{\partial r}+\frac{u_{\theta}}{r} \frac{\partial u_{\phi}}{\partial \theta}+\frac{u_{\phi}}{r \sin \theta} \frac{\partial u_{\phi}}{\partial \phi}+\frac{u_{r} u_{\phi}}{r}+\frac{u_{\theta} u_{\phi} \cot \theta}{r}+2 \Omega \cos \theta u_{\theta} \\
=-\frac{1}{r \sin \theta} \frac{\partial p}{\partial \phi}+\nu\left(\Delta u_{\phi}+\frac{2}{r^{2} \sin \theta} \frac{\partial u_{r}}{\partial \phi}+\frac{2 \cos \theta}{r^{2} \sin ^{2} \theta} \frac{\partial u_{\theta}}{\partial \phi}-\frac{u_{\phi}}{r^{2} \sin ^{2} \theta}\right) \\
\frac{1}{r^{2}} \frac{\partial}{\partial r}\left(r^{2} u_{r}\right)+\frac{1}{r \sin \theta} \frac{\partial}{\partial \theta}\left(u_{\theta} \sin \theta\right)+\frac{1}{r \sin \theta} \frac{\partial u_{\phi}}{\partial \phi}=0,
\end{gathered}
$$

where $\Omega=2 \pi \mathrm{rad} /$ day $\approx 0.73 \times 10^{-4} \mathrm{~s}^{-1}$ is the rate of the earth's rotation and

$$
\Delta=\frac{1}{r^{2}} \frac{\partial}{\partial r}\left(r^{2} \frac{\partial}{\partial r}\right)+\frac{1}{r^{2} \sin \theta} \frac{\partial}{\partial \theta}\left(\sin \theta \frac{\partial}{\partial \theta}\right)+\frac{1}{r^{2} \sin ^{2} \theta} \frac{\partial^{2}}{\partial \phi^{2}}
$$

is the Laplacian in spherical coordinates. In the above system, $r, \phi$ and $\theta$ denote the radius, azimuthal (increasing by going east) and polar (increasing by going south) angle, respectively. The associated 
velocity components are $u_{r}, u_{\phi}$ and $u_{\theta}$, respectively, and $p$ denotes the pressure and $\nu$ is kinematic viscosity.

Since the velocity vector $\vec{u}$ and the pressure $p$ are coupled together by the incompressibility constraint (1.5), $\nabla \cdot \vec{u}=0$, it is difficult to analyze the full set of three-dimensional equations (1.1) - (1.3). A common approach to simplify the nonlinear model in the question is to use artificial methods such as pressure stabilization and projection [29]. However, as has been shown in [29], the error estimate of the pressure stabilization and projection methods is not mathematically precise.

Another approach to reduce the complexity of the governing equations of geophysical fluid dynamics is to consider submodels thereof. The latter usually take into account several simplifications that make them adapted for distinct physical processes or that render them valid only on well-defined spatial or temporal scales. One of the most relevant simplifications which can be reasonably implemented in the atmospheric sciences is that of the two-dimensionality of the large-scale flow [20]; [18]. This reduction in dimensionality greatly simplifies the structure of the dynamical part of the governing equations, which can be consistently described by some forms of the two-dimensional Euler or Navier-Stokes equations [33]. Though they are still coupled systems of nonlinear partial differential equations, much is known by today about the long-term behavior of these systems.

In terms of physical interpretation, our reduction (see also [13]) consists in considering the fluid flows on a sphere of radius $r$, such that $r_{0}<|r|<r_{0}+\epsilon$, where $\epsilon$ is a nondimensional small parameter that represents the thickness of the spherical shell. Thus in the limiting case $\epsilon \rightarrow 0$, we must set $u_{r}=0$. In addition, since the atmosphere neither loses nor gains mass from outside, we naturally assume that $\left(\rho u_{r}\right) \rightarrow 0$ as $r \rightarrow \infty$, where $\rho$ is the density. However, there exists a height for which $\rho u_{r}$ attains a maximum (the other possibility corresponds to the case when the vertical velocity $u_{r}=0$ ), i.e. when

$$
\frac{\partial \rho u_{r}}{\partial r}=0
$$

It is thus postulated in the present work that at least one such level exists for the entire atmosphere of the earth. We call it the "mean level". In practice (see e.g. Refs. [6]; [7] and [4]), we can speak about it as a level of 3-5 km. Thus in the limit $\epsilon \rightarrow 0$, the non-stationary three-dimensional viscous rotating fluid flow is confined on a sphere of the radius $r_{0}$ parametrized by the polar and azimuthal angles $\theta$ and $\phi$.

In terms of rigorous mathematical treatment, the latter reduction (1.6) can also be deduced from the result of Theorem B in [33], which states that provided the initial data $\mathbf{u}(r, \theta, \phi)$ is smooth enough, the strong global solution $\mathbf{u}(r, \theta, \phi, t)$ of the three-dimensional Euler equations converges as $\epsilon \rightarrow 0$ to the strong global solution $\mathbf{v}(\theta, \phi, t)$ of the two-dimensional Euler equations on the sphere, where

$$
\mathbf{v}(\theta, \phi, t)=\lim _{\epsilon \rightarrow 0} \frac{1}{\epsilon} \int_{1}^{1+\epsilon} r \mathbf{u}(r, \theta, \phi, t) \mathrm{d} r=\left(0, u_{\theta}, u_{\phi}\right) .
$$

The vector $v(\theta, \phi, t)$ can be interpreted as the average velocity with respect to $r$.

The resulting form of the two-dimensional Euler or NS equations which is of immediate interest in large-scale atmospheric dynamics in a thin atmospheric layer on a sphere rotating with constant angular velocity $\Omega$ is

$$
\begin{gathered}
\frac{\partial v_{\theta}}{\partial t}+v_{\theta} \frac{\partial v_{\theta}}{\partial \theta}+\frac{v_{\phi}}{\sin \theta} \frac{\partial v_{\theta}}{\partial \phi}-v_{\phi}^{2} \cot \theta+R_{e}^{-1}\left(\Delta_{S} u_{\theta}+\frac{u_{\theta}-2 \cos \theta \frac{\partial u_{\phi}}{\partial \phi}}{r^{2} \sin ^{2} \theta}\right)=-\frac{\partial p}{\partial \theta} \\
\frac{\partial v_{\phi}}{\partial t}+\cot \theta v_{\theta}+v_{\theta} \frac{\partial v_{\phi}}{\partial \theta}+\frac{v_{\phi}}{\sin \theta} \frac{\partial v_{\phi}}{\partial \phi}+v_{\theta} v_{\phi} \cot \theta+ \\
R_{e}^{-1}\left(\Delta_{S} u_{\phi}+\frac{2 \cos \theta \frac{\partial u_{\theta}}{\partial \phi}-u_{\phi}}{r^{2} \sin ^{2} \theta}\right)=-\frac{1}{\sin \theta} \frac{\partial p}{\partial \phi}
\end{gathered}
$$




$$
\frac{\partial}{\partial \theta}\left(v_{\theta} \sin \theta\right)+\frac{\partial v_{\phi}}{\partial \phi}=0
$$

where $R_{e} \propto \nu^{-1}$ is the Reynolds number, $R_{0} \propto \Omega^{-1}$ is the Rossby number (which is the ratio of inertial to Coriolis force) and

$$
\Delta_{S}=\frac{1}{\sin \theta} \frac{\partial}{\partial \theta}\left(\sin \theta \frac{\partial}{\partial \theta}\right)+\frac{1}{\sin ^{2} \theta} \frac{\partial^{2}}{\partial \varphi^{2}}
$$

is the Laplace-Beltrami operator in spherical angles. The latter reduction relies on the theorem from the work [33].

We represent the solution of the model (1.8) - (1.10) in the form

$$
v_{\theta}=\mu \widehat{v}_{\theta}, \quad v_{\phi}=F(\theta)+\mu \widehat{v}_{\phi}, \quad p=\bar{p}+\mu \widehat{p},
$$

where $\mu>0$ is a (not necessarily small) parameter, $F(\theta)$ is an arbitrary function of its argument and $\bar{p}$ is the mean pressure in the thin atmospheric layer studied. Variables with hats are to be interpreted as disturbance quantities. Physically, this ansatz corresponds to finite disturbances that are superimposed to a zonally averaged mean flow having only a latitudinal dependence. Particularly, the presence of Coriolis effects originates the general west-to-east flows caused by the Earth's rotation which are related to jet streams, i.e., zones of fast-moving west-to east winds in the upper atmosphere between the Ferrell and Polar cells. Such flows exist due to the presence of the cold North and South Poles and warm Equator so that the pressure is low at high latitudes above the poles and is high in the temperate zones and even higher in the equatorial zones. Thus as has been discussed in [11] and [4], under the assumption of no friction, and a distribution of temperature dependent only upon latitude and altitude, the stationary solution (1.10) can be associated with a zonal flow directed from the west to east. The experimental results in [8] justify the conclusions on existence of such zonal flows discussed in [11].

After dropping the hat symbol, equations for disturbances are written as

$$
\begin{gathered}
\frac{\partial v_{\theta}}{\partial t}+\frac{F}{\sin \theta} \frac{\partial v_{\theta}}{\partial \phi}-2 F v_{\phi} \cot \theta+\frac{\partial p}{\partial \theta}+v_{\theta} \frac{\partial v_{\theta}}{\partial \theta}+\frac{v_{\phi}}{\sin \theta} \frac{\partial v_{\theta}}{\partial \phi}-v_{\phi}^{2} \cot \theta \\
R_{e}^{-1}\left(\Delta_{S} v_{\theta}+\frac{v_{\theta}-2 \cos \theta \frac{\partial v_{\phi}}{\partial \phi}}{\sin ^{2} \theta}\right)=0 \\
\frac{\partial v_{\phi}}{\partial t}+\left(F^{\prime}+\cot \theta\right) v_{\theta}+\frac{F}{\sin \theta} \frac{\partial v_{\phi}}{\partial \phi}+\frac{1}{\sin \theta} \frac{\partial p}{\partial \phi}+v_{\theta} \frac{\partial v_{\phi}}{\partial \theta}+\frac{v_{\phi}}{\sin \theta} \frac{\partial v_{\phi}}{\partial \phi}+v_{\theta} v_{\phi} \cot \theta \\
+R_{e}^{-1}\left(\Delta_{S} v_{\phi}+\frac{2 \cos \theta \frac{\partial v_{\theta}}{\partial \phi}-v_{\phi}}{\sin ^{2} \theta}\right)=0, \\
\frac{\partial}{\partial \theta}\left(v_{\theta} \sin \theta\right)+\frac{\partial v_{\phi}}{\partial \phi}=0
\end{gathered}
$$

where prime means differentiation and, without loss of generality, we set $\mu=1$ in front of quadratic terms.

In this work, we are mostly focused on the particular case of nonviscous flows, i.e., when $R_{e} \rightarrow \infty$. However, a discussion of a possible extension of our analysis to the case of nonzero viscosity is presented in the last section of this work.

By introducing a stream function $\psi$ for the disturbance velocities, i.e.,

$$
v_{\theta}=-\frac{1}{\sin \theta} \frac{\partial \psi}{\partial \phi}, \quad v_{\phi}=\frac{\partial \psi}{\partial \theta},
$$

and taking the curl of Eqs. (1.13) and (1.14), one arrives at the vorticity equation in the limiting case $R_{e} \rightarrow \infty$ : 


$$
\frac{\partial \zeta}{\partial t}+\frac{\mu}{\sin \theta} J\left(\frac{\partial \psi}{\partial \theta}, \frac{\partial \zeta}{\partial \phi}\right)+\frac{F}{\sin \theta} \frac{\partial \zeta}{\partial \phi}-\frac{1}{\sin \theta} \frac{\partial \psi}{\partial \phi} L_{1} F+\frac{1}{R_{0}} \frac{\partial \psi}{\partial \phi}=0,
$$

where the vorticity $\zeta$ is related to the stream function $\psi$ through the Laplacian in spherical geometry,

$$
\zeta=\Delta_{S} \psi
$$

and

$$
L_{1}=\frac{1}{\sin \theta} \frac{d}{d \theta}\left(\sin \theta \frac{d}{d \theta}\right)-\frac{1}{\sin ^{2} \theta}
$$

is the Sturm-Liouville operator for the associated Legendre functions. Additionally,

$$
J(a, b)=a_{\theta} b_{\phi}-a_{\phi} b_{\theta}
$$

stands for the nonlinear Jacobian operator with the subscripts meaning the partial differentiation.

\section{Background}

In terms of group theoretical modeling, the dynamics of the system in the question can often be described by means of the formal Lagrangian and the adjoint equations [22], [16]. The construction of conservation laws can be deduced from the Noether's theorem [27] that manifests a connection between symmetries and conservation laws for variational problems and thus provides a simpler procedure for construction of conservation laws for equations with known symmetries. Namely, our algorithm in finding the formal Lagrangian and conservation laws is based on Theorem 2.3 in [22] which states that any system (linear or nonlinear) of $s^{\text {th }}$-order of differential equations

$$
F_{\alpha}\left(x, u, u_{(1)}, \ldots, u_{(s)}\right)=0, \quad \alpha=1, \ldots, m,
$$

conserved together with its adjoint equation

$$
F_{\alpha}^{*}\left(x, u, v, \ldots, u_{(s)}, w_{(s)}\right)=\frac{\delta\left(w^{\beta} F_{\beta}\right)}{\delta u^{\alpha}}=0
$$

has a Lagrangian, where $x=\left(x^{1}, \ldots, x^{n}\right)$ be $n$ independent variables, $u=\left(u^{1}, \ldots, x^{m}\right)$ be $m$ dependent variables, $u_{(1)}, \ldots, u_{(s)}$ denote the sets of partial derivatives of the respective orders, e.g. $u_{(1)}=\left\{u_{i}^{\alpha}\right\}, u_{(2)}=\left\{u_{i j}^{\alpha}\right\}$ with

$$
u_{i}^{\alpha}=\frac{\partial u^{\alpha}(x)}{\partial x^{i}}, \quad u_{i j}^{\alpha}=\frac{\partial^{2} u^{\alpha}(x)}{\partial x^{i} \partial x^{i}}
$$

and $w=\left(w^{1}, \ldots, w^{m}\right)$ are new dependent variables. Namely, according to [22], the Lagrangian $\mathcal{L}$ is given by

$$
\mathcal{L}=w^{\beta} F_{\beta}\left(x, u, u_{(1)}, \ldots, u_{(s)}\right) .
$$

As will be outlined in sequel of this work, the nonlinear model (1.13) - (1.15) describing the dynamics of atmospheric motion in a thin rotating shell considered in Section 3 has a remarkable property to be self-adjoint for a specific choice of $F(\theta)$. This property is crucial for constructing conservation laws provided in the present study.

Although it is a common belief that the applicability of Noether's theorem is restricted because Lagrangians exist only for very special types of differential equations, it has been shown in Ref. [22] that one can associate conservation laws with symmetries of any partial differential equation using the concept (2.2) of a formal Lagrangian. 
In general, the conservation equation is written [22] as

$$
D_{t}\left(C^{1}\right)+D_{\theta}\left(C^{2}\right)+D_{\phi}\left(C^{3}\right)=0 .
$$

Particularly, the direct application of the general conservation theorem demonstrated in [22] shows that the components $C^{i}$ of the conservation law (2.4), or more concisely, $D_{i}\left(C^{i}\right)=0$, for the model in question admitting the group in the general form

$$
X=\xi^{i}(x, \psi) \frac{\partial}{\partial x^{i}}+\eta(x, \psi) \frac{\partial}{\partial \psi},
$$

can be written as

$$
\begin{aligned}
C^{i} & =W\left[\frac{\partial \mathcal{L}}{\partial \psi_{i}}-D_{j}\left(\frac{\partial \mathcal{L}}{\partial \psi_{i j}}\right)+D_{j} D_{k}\left(\frac{\partial \mathcal{L}}{\partial \psi_{i j k}}\right)\right] \\
& +D_{j}(W)\left[\frac{\partial \mathcal{L}}{\partial \psi_{i j}}-D_{k}\left(\frac{\partial \mathcal{L}}{\partial \psi_{i j k}}\right)\right]+D_{j} D_{k}(W) \frac{\partial \mathcal{L}}{\partial \psi_{i j k}},
\end{aligned}
$$

where

$$
W=\eta-\xi^{j} \psi_{j}
$$

\section{Self-adjointness and formal Lagrangian}

As will be shown in this section, the nonlinear system (1.17) describing the dynamics of atmospheric motion in a thin rotating shell has a remarkable property to be self-adjoint only for certain choice of the finite disturbances $F(\theta)$.

$$
F(\theta)=k \sin \theta
$$

where $k$ is an arbitrary constant. This choice is crucial for constructing conservation laws provided in the present study. Somewhat surprisingly, the same choice of $F$ provides the invariant (exact) solutions for the Navier-Stokes flows, i.e. when $R_{e} \neq \infty$. For investigating the self-adjointness, we write equation (1.17) in the form

$$
\Omega_{1}+\Omega_{2}+\mu \Omega_{3}=0
$$

where

$$
\begin{gathered}
\Omega_{1}=\psi_{t \theta} \cos \theta+\psi_{t \theta \theta} \sin \theta+\frac{1}{\sin \theta} \psi_{t \phi \phi}+\frac{\sin \theta}{R_{0}} \psi_{\phi}, \\
\Omega_{2}=\left(\psi_{\theta \theta \phi}+\psi_{\theta \phi} \cot \theta+\frac{1}{\sin ^{2} \theta} \psi_{\phi \phi \phi}\right) F(\theta)-\psi_{\phi} L_{1} F \\
\Omega_{3}=\left(\psi_{\theta \theta \phi}+\psi_{\theta \phi} \cot \theta+\frac{1}{\sin ^{2} \theta} \psi_{\phi \phi \phi}\right) \psi_{\theta}-\left(L_{1} \psi_{\theta}+\frac{\psi_{\theta \phi \phi}}{\sin ^{2} \theta}\right),
\end{gathered}
$$

in which the Sturm-Liouville operator $L_{1}$ for the associated Legendre functions is given by formula (1.19). Then, using notation as in Ref. [22], the adjoint equation to Equation (3.2) is written as

$$
\Omega_{1}^{*}+\Omega_{2}^{*}+\mu \Omega_{3}^{*}=0
$$

with

$$
\Omega_{1}^{*}=\frac{\delta\left(w \Omega_{1}\right)}{\delta \psi}, \quad \Omega_{2}^{*}=\frac{\delta\left(w \Omega_{2}\right)}{\delta \psi}, \quad \Omega_{3}^{*}=\frac{\delta\left(w \Omega_{3}\right)}{\delta \psi},
$$

where $w$ is a new dependent variable. By definition of the variational derivative $\delta / \delta \psi$, we have, e.g.

$$
\Omega_{1}^{*}=D_{t} D_{\theta}(w \cos \theta)-D_{t} D_{\theta}^{2}(w \sin \theta)-D_{t} D_{\phi}^{2}\left(\frac{w}{\sin \theta}\right)-D_{\phi}\left(\frac{\sin \theta}{R_{0}} w\right)
$$


and so forth. Working out the differentiations, we obtain

$$
\Omega_{1}^{*}=-\left[w_{t \theta} \cos \theta+w_{t \theta \theta} \sin \theta+\frac{1}{\sin \theta} w_{t \phi \phi}+\frac{\sin \theta}{R_{0}} w v_{\phi}\right] .
$$

It is manifest that letting here $w=\psi$ we obtain $\Omega_{1}^{*}=-\Omega_{1}$ and $\Omega_{2}^{*}=-\Omega_{2}$ holds when $w=\psi$ if and only if the function $F(\theta)$ solves the equation

$$
\frac{d F}{d \theta}=F \cot \theta,
$$

i.e., if $F(\theta)$ is given by (3.1). Finally, the reckoning shows that the equation $\Omega_{3}^{*}=-\Omega_{3}$ holds when $w=\psi$.

To summarize, Equation (1.17) is self-adjoint (i.e., the adjoint equation (3.6) coincides with equation (3.2) upon the substitution $w=\psi$ ) provided that $F(\theta)$ has the form (3.1).

Solution of the determining equations shows that the maximal symmetry group admitted by the equation (1.17) with $F(\theta)$ given by (3.1), and two parameters $\mu$ and $R_{0} \neq \infty$ is generated by the infinitedimensional Lie algebra spanned by the following operators:

$$
\begin{gathered}
X_{1}=\frac{\partial}{\partial t}, \quad X_{2}=\frac{\partial}{\partial \phi}, \quad X_{3}=\gamma(t) \frac{\partial}{\partial \psi}, \\
\left.X_{4}=2 R_{0} \mu t \frac{\partial}{\partial t}-\mu t \frac{\partial}{\partial \phi}+\left(\left[1+2 R_{0} k\right] \cos \theta\right)-2 \mu R_{0} \psi\right) \frac{\partial}{\partial \psi}, \\
X_{5}=\mu \sin \left(\phi+\frac{t}{2 R_{0}}\right) \frac{\partial}{\partial \theta}+\mu \frac{\cos \theta}{\sin \theta} \cos \left(\phi+\frac{t}{2 R_{0}}\right) \frac{\partial}{\partial \phi} \\
-\left(F(\theta)+\frac{\sin \theta}{2 R_{0}}\right) \sin \left(\phi+\frac{t}{2 R_{0}}\right) \frac{\partial}{\partial \psi}, \\
X_{6}=\mu \cos \left(\phi+\frac{t}{2 R_{0}}\right) \frac{\partial}{\partial \theta}-\mu \frac{\cos \theta}{\sin \theta} \sin \left(\phi+\frac{t}{2 R_{0}}\right) \frac{\partial}{\partial \phi} \\
-\left(F(\theta)+\frac{\sin \theta}{2 R_{0}}\right) \cos \left(\phi+\frac{t}{2 R_{0}}\right) \frac{\partial}{\partial \psi},
\end{gathered}
$$

where $\gamma(t)$ in the operator $X_{3}$ is an arbitrary function.

For the purpose of our work it is convenient to rewrite equation (1.17) in the form

$$
\begin{gathered}
\Lambda=\left[\psi_{t \theta} \cos \theta+\psi_{t \theta \theta} \sin \theta+\frac{1}{\sin \theta} \psi_{t \phi \phi}\right]+ \\
\left(\frac{1}{R_{0}}+2 k\right) \psi_{\phi} \sin \theta+k\left(\psi_{\theta \phi} \cos \theta+\psi_{\theta \theta \phi} \sin \theta+\frac{\psi_{\phi \phi \phi}}{\sin \theta}\right)+ \\
\mu\left(\cot \theta \psi_{\theta \phi}+\psi_{\theta \theta \phi}+\frac{\psi_{\phi \phi \phi}}{\sin ^{2} \theta}\right) \psi_{\theta}- \\
\mu\left(\cot \theta \psi_{\theta \theta}+\psi_{\theta \theta \theta}+\frac{\psi_{\theta \phi \phi}-\psi_{\theta}}{\sin ^{2} \theta}-\frac{2 \cos \theta}{\sin ^{3} \theta}\right) \psi_{\phi}=0 .
\end{gathered}
$$

Recall that $\mathrm{L}$ is the formal Lagrangian for equation for (3.13) and that it should be written in the symmetric form which, for the case of arbitrary $F(\theta)$, was calculated in [17] as

$$
\mathcal{L}=v\left[\frac{1}{2} \psi_{t \theta} \cos \theta+\frac{1}{2} \psi_{\theta t} \cos \theta+\frac{1}{3} \psi_{t \theta \theta} \sin \theta+\frac{1}{3} \psi_{\theta t \theta} \sin \theta+\frac{1}{3} \psi_{\theta \theta t} \sin \theta\right.
$$




$$
\begin{gathered}
+\frac{\psi_{t \phi \phi}}{3 \sin \theta}+\frac{\psi_{\phi t \phi}}{3 \sin \theta}+\frac{\psi_{\phi \phi t}}{3 \sin \theta}+\frac{\sin \theta}{R_{0}} \psi_{\phi}+ \\
\left(\frac{\cot \theta \psi_{\theta \phi}}{2}+\frac{\cot \theta \psi_{\phi \theta}}{2}+\frac{\psi_{\theta \theta \phi}}{3}+\frac{\psi_{\theta \phi \theta}}{3}+\frac{\psi_{\phi \theta \theta}}{3}+\frac{\psi_{\phi \phi \phi}}{\sin ^{2} \pi}\right)\left[F(\theta)-\mu \psi_{\theta}\right]-L F(\theta) \psi_{\phi}- \\
\left.\mu\left(\cot \theta \psi_{\theta \theta}+\psi_{\theta \theta \theta}-\frac{\psi_{\theta}}{\sin ^{2} \theta}-\frac{2 \cos \theta}{\sin ^{3} \theta} \psi_{\phi \phi}+\frac{\psi_{\theta \phi \phi}}{3 \sin ^{2} \theta}+\frac{\psi_{\phi \theta \phi}}{3 \sin ^{2} \theta}+\frac{\psi_{\phi \phi \theta}}{3 \sin ^{2} \theta}\right) \psi_{\phi}\right] .
\end{gathered}
$$

Since we are interested in the nonlinearly self-adjoint case, we now consider the case when $F(\theta)$ has the form (3.1). We let $v=\psi$ due to the self-adjointness of equation (1.17) and obtain

$$
\begin{gathered}
\mathcal{L}=\psi\left[\frac{\cos \theta}{2}\left(\psi_{t \theta}+\psi_{\theta t}\right)+\frac{\sin \theta}{3}\left(\psi_{t \theta \theta}+\psi_{\theta t \theta}+\psi_{\theta \theta t}\right)\right. \\
+\left(\frac{1}{R_{0}}+2 k\right) \psi_{\phi} \sin \theta+\frac{k \cos \theta}{2}\left(\psi_{\theta \phi}+\psi_{\phi \theta}\right)+\frac{\psi_{t \phi \phi}+\psi_{\phi t \phi}+\psi_{\phi \phi t}}{3 \sin \theta}+ \\
\frac{k \sin \theta}{3}\left(\psi_{\theta \theta \phi}+\psi_{\theta \phi \theta}+\psi_{\phi \theta \theta}\right)+\frac{k}{\sin \theta} \psi_{\phi \phi \phi}+ \\
\mu \psi_{\theta}\left(\frac{\cot \theta\left(\psi_{\theta \phi}+\psi_{\phi \theta}\right)}{2}+\frac{1}{3}\left(\psi_{\theta \theta \phi}+\psi_{\theta \phi \theta}+\psi_{\theta \phi \phi}\right)+\frac{\psi_{\phi \phi \phi}}{\sin ^{2} \theta}\right)- \\
\left.\mu \psi_{\phi}\left(\cot \theta \psi_{\theta \theta}+\psi_{\theta \theta \theta}-\frac{\psi_{\theta}}{\sin ^{2} \theta}-\frac{2 \cos \theta}{\sin ^{3} \theta} \psi_{\phi \phi}+\frac{\psi_{\theta \phi \phi}+\psi_{\phi \theta \phi+} \psi_{\phi \phi \theta}}{3 \sin ^{2} \theta}\right)\right] .
\end{gathered}
$$

\section{Calculation of conserved vectors}

We calculate in this section all conserved vectors associated with the Lie point symmetries of equation (1.17). Using notation $x^{1}=t, x^{2}=\theta, x^{3}=\phi$, we write the conservation law for the vector (2.6) in the form (2.4). The conserved vectors have been computed using the package MAPLE.

\subsection{Conserved vector associated with $X_{1}$}

We begin with finding all components $C^{1}, C^{2}$ and $C^{3}$ of the conservation equation (??) associated with the symmetry $X_{1}$. Following notation in Ref. [22], the component $C^{1}$ of the conserved vector is referred to as the conserved density. Here $W=-\psi_{t}$ and the computation gives the conserved vector with the components

$$
C_{X_{1}}^{1}=\psi_{\theta} \psi_{t \theta} \sin \theta+\frac{1}{\sin \theta} \psi_{\phi} \psi_{t \phi},
$$

I

$$
\begin{gathered}
C_{X_{1}}^{2}=-\left(\psi_{t} \psi_{t \theta}+\psi \psi_{t t \theta}+k \psi_{t} \psi_{\theta \phi}+k \psi \psi_{t \theta \phi}\right) \sin \theta+ \\
\mu\left[\psi \psi_{\phi} \psi_{t \theta \theta}-\psi \psi_{t} \psi_{\theta \theta \phi}+\cot \theta\left(\psi \psi_{\phi} \psi_{t \theta}-\psi \psi_{t} \psi_{\theta \phi}\right)+\right. \\
\left.\frac{1}{\sin ^{2} \theta}\left(\psi \psi_{\phi} \psi_{t \phi \phi}-\psi \psi_{t} \psi_{\phi \phi \phi}\right)\right] \\
C_{X_{1}}^{3}=-\frac{\sin \theta}{R_{0}} \psi \psi_{t}-\frac{1}{\sin \theta}\left(\psi_{t} \psi_{t \phi}+\psi \psi_{t t \phi}\right)- \\
k\left[\left(2 \psi \psi_{t}-\psi_{\theta} \psi_{t \theta}\right) \sin \theta+\frac{1}{\sin \theta}\left(\psi_{t} \psi_{\phi \phi}-\psi_{\phi} \psi_{t \phi}+\psi \psi_{t \phi \phi}\right)\right]+ \\
\mu\left[\psi \psi_{t} \psi_{\theta \theta \theta}-\psi \psi_{\theta} \psi_{t \theta \theta}+\frac{1}{\sin \theta}\left(\psi \psi t \psi_{\theta \theta}-\psi \psi_{\theta} \psi_{t \theta}\right)+\right.
\end{gathered}
$$




$$
\left.\frac{1}{\sin ^{2} \theta}\left(\psi \psi_{t} \psi_{\theta \phi \phi}-\psi \psi_{\theta} \psi_{t \phi \phi}-\psi \psi_{t} \psi_{\theta}\right)-\frac{2 \cos \theta}{\sin ^{3} \theta} \psi \psi_{t} \psi_{\phi \phi}\right]
$$

It is shown in [17] that the conserved density (4.1) is trivial, namely it can be written in the divergent form

$$
C_{X_{1}}^{1}=-\psi \Lambda+D_{\theta}(\Theta)+D_{\phi}(\Phi)
$$

where $\Lambda$ is defined in equation (3.13) and

$$
\begin{gathered}
\Theta=\psi \psi_{t \theta} \sin \theta+k \psi \psi_{\theta \phi} \sin \theta-\mu\left(\psi \psi_{\phi} \psi_{\theta \theta}+\cot \theta \psi \psi_{\theta} \psi_{\phi}-\frac{\psi_{\phi}^{3}}{2 \sin ^{2} \theta}\right), \\
\Phi=\frac{\psi \psi_{t \phi}}{\sin \theta}+\left(\frac{1}{2 R_{0}}+k\right) \psi^{2} \sin \theta+\frac{k}{2 \sin \theta}\left(2 \psi \psi_{\phi \phi}-\psi_{\phi}^{2}\right)-\frac{k}{2} \psi_{\theta}^{2} \sin \theta+ \\
\mu\left[\psi \psi_{\theta} \psi_{\theta \theta}+\frac{1}{2 \sin ^{2} \theta}\left(2 \psi \psi_{\theta} \psi_{\phi \phi}-2 \psi \psi_{\phi} \psi_{\theta \phi}-\psi_{\theta} \psi_{\phi}^{2}\right)+\cot \theta \psi \psi_{\theta}^{2}+\frac{\cos \theta}{\sin ^{3} \theta} \psi \psi_{\phi}^{2}\right] .
\end{gathered}
$$

Therefore one can nullify $C^{1}$ by transferring the terms $D_{\theta}(\Theta)$ and $D_{\phi}(\Phi)$ into $C_{X_{1}}^{2}$ and $C_{X_{1}}^{3}$, respectively. As a result, we first obtain

$$
\begin{gathered}
\widetilde{C}_{X_{1}}^{2}=C_{X_{1}}^{2}+D_{t}(\Theta)=D_{\phi}(\mu \widetilde{\Phi}), \\
\widetilde{C}_{X_{1}}^{3}=C_{X_{1}}^{3}+D_{t}(\Phi)=D_{\theta}(-\mu \widetilde{\Phi}),
\end{gathered}
$$

where

$$
\widetilde{\Phi}=-\psi \psi_{t} \psi_{\theta \theta}-\cot \theta \psi \psi_{t} \psi_{\theta}+\frac{1}{2 \sin ^{2} \theta}\left(2 \psi \psi_{\phi} \psi_{t \phi}-2 \psi \psi_{t} \psi_{\phi \phi}+\psi_{t} \psi_{\phi}^{2}\right)
$$

It follows that the vector $(4.1)$ - (4.3) is equivalent to the trivial conserved vector

$$
\widetilde{C}=(-\psi \Lambda, 0,0) .
$$

\subsection{Conserved vector associated with $X_{2}$}

For the symmetry $X_{2}$ from (3.9) we obtain

$$
\begin{gathered}
C_{X_{2}}^{1}=-\psi_{\phi}\left(D_{\theta}\left(\psi_{\theta} \sin \theta\right)+\frac{\psi_{\phi \phi}}{\sin \theta}\right)= \\
D_{\phi}\left[\frac{1}{2} \psi_{\theta}^{2} \sin \theta-\frac{\psi_{\phi}^{2}}{2 \sin \theta}\right]-D_{\theta}\left(\psi_{\phi} \psi_{\theta} \sin \theta\right) .
\end{gathered}
$$

Calculating further we see that the conserved vector has the form

$$
\begin{gathered}
C_{X_{2}}^{1}=0, \\
C_{X_{2}}^{2}=D_{\phi}(\Phi), \\
C_{X_{2}}^{3}=-D_{\theta}(\Phi)-\psi \Lambda,
\end{gathered}
$$

where

$$
\begin{gathered}
\Phi=\mu\left(\frac{\psi_{\phi}^{3}-\psi_{\phi \phi} \psi_{\phi} \psi}{3 \sin ^{2} \theta}-\frac{\cos \theta \psi \psi_{\theta} \psi_{\phi}}{2 \sin \theta}+\frac{\psi \psi_{\phi} \psi_{\theta \theta}}{3}+\frac{\psi_{\theta}^{2} \psi_{\phi}}{3}-\frac{2 \psi \psi_{\theta} \psi_{\theta \phi}}{3}\right)- \\
\frac{k}{6} \psi \psi_{\phi} \cos \theta+\frac{1}{3}\left(k \psi_{\theta} \psi_{\phi}-2 k \psi \psi_{\theta \phi}-3 \psi \psi_{t \theta}\right) \sin \theta
\end{gathered}
$$

Hence the symmetry $X_{2}$ provides the trivial conserved vector

$$
(0,0,-\psi \Lambda) \text {. }
$$




\subsection{Conserved vector associated with $X_{3}$}

For the symmetry $X_{3}$ we obtain the following nontrivial conserved vector:

$$
\begin{gathered}
C_{X_{3}}^{1}=0, \\
C_{X_{3}}^{2}=\gamma(t)\left[\mu\left(-\frac{\psi_{\phi} \psi_{\phi \phi}}{\sin ^{2} \theta}-\psi_{\phi} \psi_{\theta \theta}-\cot \theta \psi_{\theta} \psi_{\phi}\right)+\psi_{t \theta} \sin \theta+k \psi_{\theta \phi} \sin \theta\right], \\
C_{X_{3}}^{3}=\gamma(t)\left[\mu\left(\frac{\psi_{\theta} \psi_{\phi \phi}}{\sin ^{2} \theta}+\psi_{\theta} \psi_{\theta \theta}+\cot \theta \psi_{\theta}^{2}\right)+\frac{1}{\sin \theta}\left(\psi_{t \phi}+k \psi_{\phi \phi}\right)+\left(\frac{1}{R_{0}}+2 k\right) \psi \sin \theta\right] .
\end{gathered}
$$

For this vector the conservation equation is satisfied in the form

$$
D_{t}\left(C_{X_{3}}^{1}\right)+D_{\theta}\left(C_{X_{3}}^{2}\right)+D_{\phi}\left(C_{X_{3}}^{3}\right)=\gamma(t) \Lambda .
$$

\subsection{Conserved vector associated with $X_{4}$}

The symmetry $X_{4}$ provides a nontrivial conserved vector. Namely,

$$
C_{X_{4}}^{1}=\left[\sin \theta\left(1+2 R_{0} k\right) v_{\phi}+2 \mu R_{0}\left(v_{\theta}^{2}+v_{\phi}^{2}\right)+\mu t R_{0}\left(v_{\theta}^{2}+v_{\phi}^{2}\right)_{t}\right] \sin \theta,
$$

The conserved density (4.8) can be reduced to the form

$$
C_{X_{4}}^{1}=\left(1+2 R_{0} k\right) \psi_{\theta}^{2} \sin ^{2} \theta+2 \mu R_{0}\left(\psi_{\theta}^{2} \sin \theta+\frac{1}{\sin \theta} \psi_{\phi}^{2}\right)
$$

because

$$
\mu t R_{0}\left(v_{\theta}^{2}+v_{\phi}^{2}\right)_{t} \sin \theta=2 \mu t R_{0}\left[D_{\theta}(t \Theta)+D_{\phi}(t \Phi)-t \psi \Lambda\right],
$$

where $\Theta$ and $\Phi$ are the same as in equation (4.4). For $C_{X_{4}}^{2}$ and $C_{X_{4}}^{3}$ we obtain

$$
\begin{gathered}
C_{X_{4}}^{2}=4 \mu^{2} R_{0} \psi\left(\psi_{\phi} \psi_{\theta \theta}+\cot \theta \psi_{\theta} \psi_{\phi \phi}\right)- \\
\mu\left[4 R_{0} \psi\left(k \psi_{\theta \phi}+\psi_{t \theta}\right) \sin \theta+\left(2 R_{0} k+1\right)\left(\frac{\psi_{\theta} \psi_{\phi}}{\sin \theta}+\psi_{\phi} \psi_{\theta \theta} \cos \theta\right)\right]+\left(2 R_{0} k+1\right)\left(\psi_{t \theta \phi} \sin \theta \cos \theta\right), \\
C_{X_{3}}^{4}=-4 \mu^{2} R_{0} \psi\left(\psi_{\theta} \psi_{\theta \theta}+\cot \theta \psi_{\theta}^{2}+\frac{\psi_{\theta} \psi_{\phi \phi}}{\sin ^{2} \theta}\right)+ \\
\mu\left[-\frac{4 R_{0}}{\sin \theta}\left(k \psi_{\phi \phi}+\psi_{t \phi}\right) \psi+\right. \\
\left(2 R_{0} k+1\right)\left(\frac{\psi_{\phi}^{2}}{\sin ^{3} \theta}+\psi_{\theta} \psi_{\theta \theta} \cos \theta+\frac{\cos \theta}{\sin { }^{2} \theta}\left(\psi_{\theta} \psi_{\phi \phi}-\psi_{\phi} \psi_{\theta \phi}\right)+\frac{\psi_{\theta}^{2}}{\sin \theta}-2 \psi^{2} \sin \theta\right)- \\
\left.\frac{\psi_{\phi}^{2}}{\sin \theta}+\frac{1}{2}\left(2 R_{0} k-1\right) \psi_{\theta}^{2} \sin \theta\right]+ \\
\left(2 R_{0} k+1\right)\left[k \psi_{\theta}\left(1-\sin ^{2} \theta\right)+\cot \theta\left(k \psi_{\phi \phi}+\psi_{t \phi}\right)+\left(\left(2 k+\frac{1}{R_{0}}\right) \psi+k \psi_{\theta \theta}\right) \sin \theta \cos \theta\right] .
\end{gathered}
$$

In this case the conservation equation (2.4) has the form

$$
D_{t}\left(C_{X_{4}}^{1}\right)+D_{\theta}\left(C_{X_{4}}^{2}\right)+D_{\phi}\left(C_{X_{4}}^{3}\right)=\left(\left(2 k R_{0}+1\right) \cos \theta-4 \mu R_{0} \psi\right) \Lambda .
$$




\subsection{Conserved vector associated with $X_{5}$}

Considering $X_{5}$ we obtain the conserved vector

$$
C_{X_{5}}^{1}=\left(k+\frac{1}{2 R_{0}}\right)\left(\psi_{\theta} \sin \theta \cos \theta-\psi_{\phi \phi}\right) \sin \tau,
$$

The conserved density (4.8) can be reduced to the form

$$
\begin{gathered}
C_{X_{5}}^{2}=\mu^{2}\left(\psi_{\phi} \cos \tau+\psi \sin \tau\right)\left[-\frac{2 \cos \theta}{\sin ^{3} \theta} \psi_{\phi} \psi_{\phi \phi}+\frac{1}{\sin ^{2} \theta}\left(\psi_{\phi} \psi_{\theta \phi \phi}-\psi_{\theta} \psi_{\phi}-\psi_{\theta} \psi_{\phi \phi \phi}\right)+\right. \\
\left.\cot \theta\left(\psi_{\phi} \psi_{\theta \theta}-\psi_{\theta} \psi_{\theta \phi}\right)+\psi_{\phi} \psi_{\theta \theta \theta}-\psi_{\theta} \psi_{\theta \theta \phi}\right]+ \\
\mu\left(k+\frac{1}{2 R_{0}}\right)\left(\frac{2}{\sin \theta} \psi_{\phi} \psi_{\phi \phi}+\left(\psi_{\phi} \psi_{\theta \theta}-\psi_{\theta} \psi_{\theta \phi}\right) \sin \theta\right) \sin \tau-\mu\left(\psi_{\phi} \cos \tau+\psi \sin \tau\right)\left[\frac{1}{\sin \theta}\left(k \psi_{\phi \phi \phi}+\psi_{t \phi \phi}\right)+\right. \\
\left.\left(\left(k+\frac{1}{2 R_{0}}\right) 2 \psi_{\phi}+k \psi_{\theta \theta \phi}+\psi_{t \theta \theta}\right) \sin \theta+\left(k \psi_{\theta \phi}+\psi_{t \theta}\right) \cos \theta\right]+ \\
\sin \theta\left(k+\frac{1}{2 R_{0}}\right)\left[k \psi_{\phi} \cos \theta \sin \tau-\left(k \psi_{\theta \phi}+\psi_{t \theta}\right) \sin \theta \sin \tau-\frac{1}{2 R_{0}} \psi \cos \theta \cos \tau\right] \\
C_{X_{5}}^{3}=\frac{\mu}{\sin \theta}\left(k+\frac{1}{2 R_{0}}\right)\left[\psi_{\theta} \psi_{\phi} \cos \tau-\left(\psi_{\phi} \psi_{\theta \phi}+\psi_{\theta} \psi_{\phi \phi}\right) \sin \tau\right]+ \\
\left(k+\frac{1}{2 R_{0}}\right)^{2} \psi_{\phi} \cos \tau+\left(k+\frac{1}{2 R_{0}}\right)\left(\frac{\psi}{2 R_{0}}\left(1-2 \sin ^{2} \theta\right)-k \psi_{\phi \phi}\right) \sin \tau
\end{gathered}
$$

where

$$
\tau=\phi+\frac{t}{2 R_{0}} .
$$

The conservation equation (2.4) has the form

$$
\begin{gathered}
D_{t}\left(C_{X_{5}}^{1}\right)+D_{\theta}\left(C_{X_{5}}^{2}\right)+D_{\phi}\left(C_{X_{5}}^{3}\right)=-\mu\left(\psi \sin \tau+\psi_{\phi} \cos \tau\right) D_{\theta}(\Lambda)- \\
{\left[\mu\left(\psi_{\theta} \sin \tau+\psi_{\theta \phi} \cos \tau\right)+\left(k+\frac{1}{2 R_{0}}\right) \sin \theta \sin \tau\right] \Lambda}
\end{gathered}
$$

\subsection{Conserved vector associated with $X_{6}$}

Finally, using the symmetry $X_{6}$ we obtain the similar conserved vector:

$$
C_{X_{6}}^{1}=\left(k+\frac{1}{2 R_{0}}\right)\left(\psi_{\theta} \sin \theta \cos \theta-\psi_{\phi \phi}\right) \cos \tau,
$$

The conserved density (4.8) can be reduced to the form

$$
\begin{gathered}
C_{X_{6}}^{2}=\mu^{2}\left(\psi_{\phi} \sin \tau-\psi \cos \tau\right)\left[\frac{2 \cos \theta}{\sin ^{3} \theta} \psi_{\phi} \psi_{\phi \phi}+\frac{1}{\sin ^{2} \theta}\left(\psi_{\theta} \psi_{\phi}-\psi_{\phi} \psi_{\theta \phi \phi}+\psi_{\theta} \psi_{\phi \phi \phi}\right)+\right. \\
\left.\cot \theta\left(\psi_{\theta} \psi_{\theta \phi}-\psi_{\phi} \psi_{\theta \theta}\right)+\psi_{\theta} \psi_{\theta \theta \phi}-\psi_{\phi} \psi_{\theta \theta \theta}\right]+ \\
\mu\left(k+\frac{1}{2 R_{0}}\right)\left(\frac{2}{\sin \theta} \psi_{\phi} \psi_{\phi \phi}+\left(\psi_{\phi} \psi_{\theta \theta}-\psi_{\theta} \psi_{\theta \phi}\right) \sin \theta\right) \cos \tau+\mu\left(\psi_{\phi} \sin \tau-\psi \cos \tau\right)\left[\frac{1}{\sin \theta}\left(k \psi_{\phi \phi \phi}+\psi_{t \phi \phi}\right)+\right. \\
\left.\left(\left(k+\frac{1}{2 R_{0}}\right) 2 \psi_{\phi}+k \psi_{\theta \theta \phi}+\psi_{t \theta \theta}\right) \sin \theta+\left(k \psi_{\theta \phi}+\psi_{t \theta}\right) \cos \theta\right]+
\end{gathered}
$$




$$
\begin{gathered}
\sin \theta\left(k+\frac{1}{2 R_{0}}\right)\left[\frac{1}{2 R_{0}} \psi \cos \theta \sin \tau+k \psi_{\phi} \cos \theta \cos \tau-\left(k \psi_{\theta \phi}+\psi_{t \theta}\right) \sin \theta \cos \tau\right], \\
C_{X_{6}}^{3}=-\frac{\mu}{\sin \theta}\left(k+\frac{1}{2 R_{0}}\right)\left[\psi_{\theta} \psi_{\phi} \sin \tau+\left(\psi_{\phi} \psi_{\theta \phi}+\psi_{\theta} \psi_{\phi \phi}\right) \cos \tau\right]- \\
\left(k+\frac{1}{2 R_{0}}\right)^{2} \psi_{\phi} \sin \tau+\left(k+\frac{1}{2 R_{0}}\right)\left(\frac{\psi}{2 R_{0}}\left(1-2 \sin ^{2} \theta\right)-k \psi_{\phi \phi}\right) \cos \tau,
\end{gathered}
$$

where $\tau$ is defined by (4.11).

Correspondingly, the conservation equation (2.4) has the form

$$
\begin{gathered}
D_{t}\left(C_{X_{6}}^{1}\right)+D_{\theta}\left(C_{X_{6}}^{2}\right)+D_{\phi}\left(C_{X_{6}}^{3}\right)=\mu\left(\psi_{\phi} \sin \tau-\psi \cos \tau\right) D_{\theta}(\Lambda)+ \\
{\left[\mu\left(\psi_{\theta \phi} \sin \tau-\psi_{\theta} \cos \tau\right)-\left(k+\frac{1}{2 R_{0}}\right) \sin \theta \sin \tau\right] \Lambda .}
\end{gathered}
$$

\section{Applications}

As an illustration to our calculations, the remainder of this article is devoted to discussion and visualization of the particular conserved densities $C_{X_{1}}^{1}$ and $C_{X_{4}}^{1}$ given by (4.1) and (4.8) respectively. As shown in our recent work in Ref. [15], the two exact nonstationary solutions for the nonlinear model (1.17) in the limiting case $R_{e}=\infty$ (non-viscous fluid) can be written in terms of the stream function $\psi$ for an arbitrary disturbance $F(\theta)$ as

$$
\begin{aligned}
\psi^{[1]} & =\frac{\cos \theta}{2 \mu R_{o}}-\frac{H(\theta)}{\mu}+\frac{c_{1}}{t}+\frac{c_{2}}{t} \ln \left|\tan \frac{\theta}{2}\right|, \\
\psi^{[2]} & =\frac{\cos \theta}{2 \mu R_{o}}-\frac{H(\theta)}{\mu}+\frac{1}{t} \Phi(\lambda),
\end{aligned}
$$

where

$$
\lambda=\sin \theta \cos \tau \text { and } \Phi(\lambda)=c_{1}+c_{2} \ln \frac{1-\lambda}{1+\lambda},
$$

in which $\tau$ is given by (4.11) and $c_{1}$ and $c_{2}$ are constants that are determined from the boundary conditions. Namely, as has been discussed in Ref. [13] the solution for the truncated spherical layer

$$
S_{0}=\left\{(\theta, \phi): \theta_{0} \leq \theta \leq \pi-\theta_{0}, 0 \leq \phi \leq 2 \pi\right\},
$$

were defined on the closed interval $\theta_{0}<\theta \leqslant \pi-\theta_{0}$ for $\theta<\theta_{0}<\pi / 2$ is subject to the boundary conditions $\psi\left(\theta_{0}\right)=\psi^{\prime}\left(\theta_{0}\right)=\psi\left(\pi-\theta_{0}\right)=\psi^{\prime}\left(\pi-\theta_{0}\right)=0$ meaning that components of the velocity vector vanish at the regular end points of the domain. Since $\theta=0$ and $\theta=\pi$ are singular points of the interval $0<\theta \leqslant \pi$, the solution $\psi(\theta)$ for the complete sphere with the singular end points is defined on an open interval $0<\theta<\pi$ satisfying the boundary conditions $\lim _{\theta \rightarrow 0} \psi(\theta)=\lim _{\theta \rightarrow \pi} \psi(\theta)=\lim _{\theta \rightarrow 0} \sin \theta \psi^{\prime}(\theta)=\lim _{\theta \rightarrow \pi} \sin \psi^{\prime}(\theta)=0$ meaning that the components of the vorticity

$$
\frac{\partial\left(\sin \theta v_{\phi}\right)}{\partial \theta}-\frac{\partial v_{\theta}}{\partial \phi}
$$

vanish at the singular end points of the domain. Without loss of generality, the spherical layer $S_{0}$ is truncated symmetrically at the two rings located in the northern and southern hemispheres so that the velocity components and pressure terms associated with the invariant solutions (5.1) and (5.2) are free of pole singularities in $S_{0}$. Additionally, for the purpose of visualization, the singularities of the function $\Phi(\lambda)$ and its derivatives were removed artificially by replacing the values $\lambda=1$ by $\lambda=0.999$. Also, since 
we are interested in nonlinear atmospheric modeling, without loss of generality, we further set $\mu=1$. We remark that if $F(\theta)$ is given by (3.1) with $k=-1 / 2 R_{0}$, i.e. if

$$
F=-\frac{\sin \theta}{2 R_{0}}
$$

then the both invariant solutions $\psi^{[1]}$ and $\psi^{[2]}$ given by (5.1) and (5.2) of the Euler equations (i.e., when $R_{e}=\infty$ ) are also exact solutions of two-dimensional Navier-Stokes equations (i.e. when $R_{e} \neq \infty$ ). The latter fact follows from direct calculations. Namely, one can check by direct differentiating that if $F$ is given by (5.5), then the both solutions $\psi^{[1]}$ and $\psi^{[2]}$ are also the solutions of the coupled equations

$$
\begin{aligned}
& \Delta_{S} v_{\theta}-\frac{v_{\theta}}{\sin ^{2} \theta}-\frac{2 \cos \theta}{\sin ^{2} \theta} \frac{\partial v_{\phi}}{\partial \phi}=0, \\
& \Delta_{S} v_{\phi}-\frac{v_{\phi}}{\sin ^{2} \theta}+\frac{2 \cos \theta}{\sin ^{2} \theta} \frac{\partial v_{\theta}}{\partial \phi}=0,
\end{aligned}
$$

which represent the "viscous corrections" in equations (1.13) and (1.14) in the case when $R_{e} \neq \infty$.

We further consider the particular case when $F$ is given by (5.5), for which the corresponding velocity components $\left(v_{\theta}^{[i]}, v_{\phi}^{[i 1]}\right)$ associated with the stream function $\psi^{[i]}(i=1,2)$ are

$$
\begin{gathered}
v_{\theta}^{[1]}=0 ; \quad v_{\phi}^{[1]}=\frac{c_{2}}{t \sin \theta}, \\
v_{\theta}^{[2]}=-\frac{2 c_{2}}{t\left(1-\lambda^{2}\right)} \sin \left(\phi+\frac{t}{2 R_{0}}\right) ; \quad v_{\phi}^{[2]}=-\frac{c_{2}}{1-\lambda^{2}} \cos \theta \cos \left(\phi+\frac{t}{2 R_{0}}\right) .
\end{gathered}
$$

For the purpose of visualization of the conserved densities associated with the invariant solutions $\psi^{[1]}$ and $\psi^{[2]}$, the color ranges in Figures 1 and 3 were manually selected to avoid extreme values near the polar caps that would cause the plots to appear uniform. In actuality, these manually selected color ranges correspond to a condensed subset of data. By using these condensed ranges, the visualizations are better able to convey the dynamical behavior of the model, which is the main purpose of those respective figures. The value $R_{0}=68.4932$ has been chosen for the following reason; for a typical atmosphere, we can choose the characteristic velocity and length scale to be $c_{0} \sim 10 \mathrm{~m} / \mathrm{s}$, and $r_{0} \sim 10^{3}$ (see also Table I in Ref. [35]) so that we have $1 / R_{o} \approx 0.0146$. A small Rossby number $R_{0}$ signifies a system which is strongly affected by Coriolis force while a large Rossby number signifies a system in which inertial and centrifugal forces dominate. As is seen from the analysis in this work, our model is related to the second situation of the large Rossby numbers.

Figure 1 illustrates the conserved quantity $C_{X_{1}}^{1,[i]}(i=1,2)$ on the truncated spherical surface $S_{0}$ corresponding to the invariant solutions $\psi^{[1]}$ (upper panel) and $\psi^{[2]}$ (lower panel), given by (5.1) and (5.2). These densities were plotted at different increasing values of time, $t$, to show their convergent behavior. Figure 2 is used to illustrate the convergent behavior of $C_{X_{1}}^{1,[1]}$ and $C_{X_{1}}^{1,[2]}$ and is comprised of twodimensional plots of the latter conserved densities vs $\theta$ at the same values of time used in Figure 1 . The convergence of $C_{X_{1}}^{1,[1]}$ and $C_{X_{1}}^{1,[2]}$ is manifested in Figure 2 by comparing the scales, e.g. in panels (a) and (d). Note that for the spherical plot in Figure 1 and all spherical plots that follow, the color scale has been manually selected to produce a clear visualization of the behavior of the solutions.

In contrast with $C_{X_{1}}^{1,[i]}$, we observe the divergent behavior of the conserved density $C_{X_{4}}^{1,[i]}$ as shown in Figures 3 and 4 . It is also remarkable that the density $C_{X_{4}}^{1,[1]}$ is a steady state solution (and actually $C_{X_{4}}^{1,[1]}$ $=0$ ) for the given choice of $F(\theta)$, which is clearly shown in the first row of spheres in Figure 3 and the blue circles in the subplots of Figure 4 . We also observe that the conserved density $C_{X_{4}}^{1,[2]}$ diverges from this steady state solution with increasing time. 


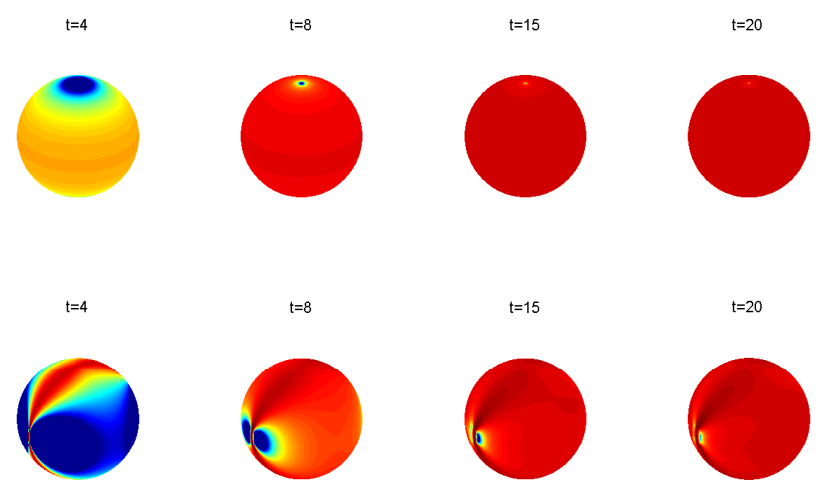

FIGURE 1. Snapshot showing the asymptotic convergence of the concerved density $C_{X_{1}}^{1,[1]}$ associated with the first invariant solution $\psi^{[1]}$ (upper panel) to the energy density $C_{X_{1}}^{1,[2]}$ associated with the second invariant solution $\psi^{[2]}$ (lower panel) plotted on the truncated spherical surface $S_{0}$ at different values of time $t$ for the case.

$$
C_{X_{1}^{1}}^{1,[i]}(i=1,2)
$$
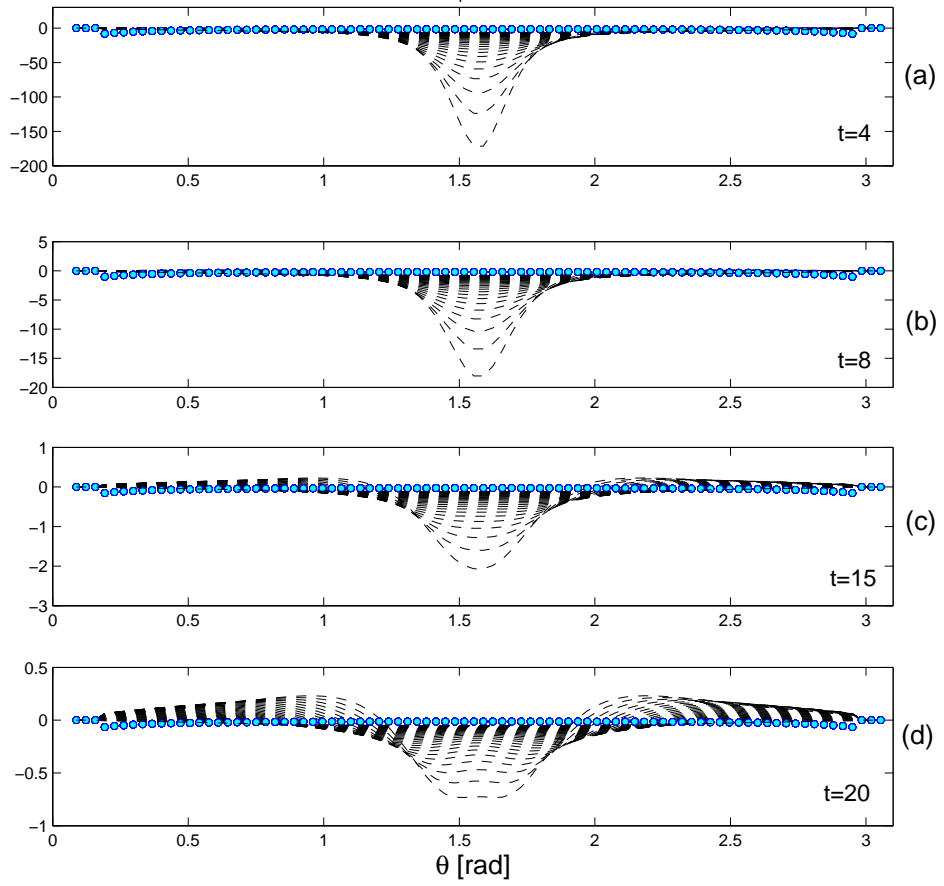

Figure 2. Asymptotic convergence of the densities $C_{X_{1}}^{1,[2]}$ (black dashed lines) and $C_{X_{1}}^{1,[1]}$ (blue circles) shown as a two-dimensional plot versus $\theta$ for several values of $\phi \in(0,2 \pi)$. 


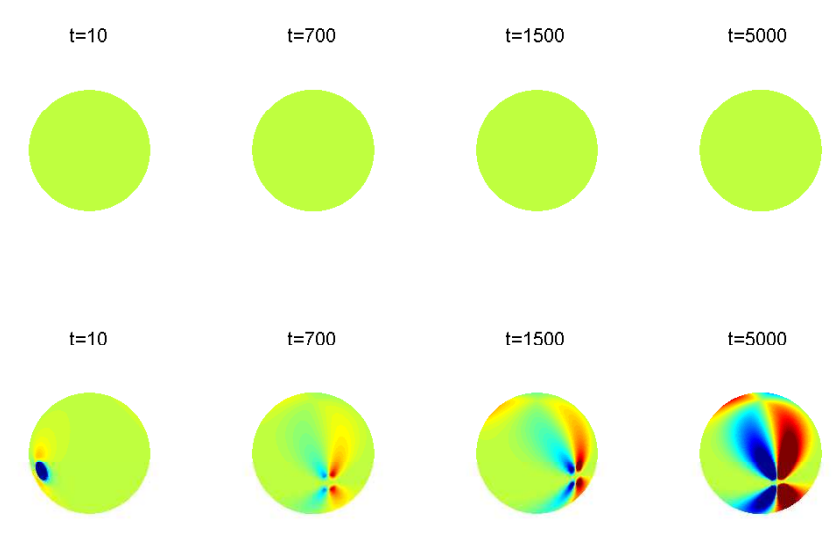

FIgURE 3. Snapshot showing the asymptotic divergence of the densities $C_{X_{4}}^{1,[1]}$ (upper panel) and $C_{X_{4}}^{1,[2]}$ (lower panel) plotted on the truncated spherical surface $S_{0}$ at different values of time $t$.
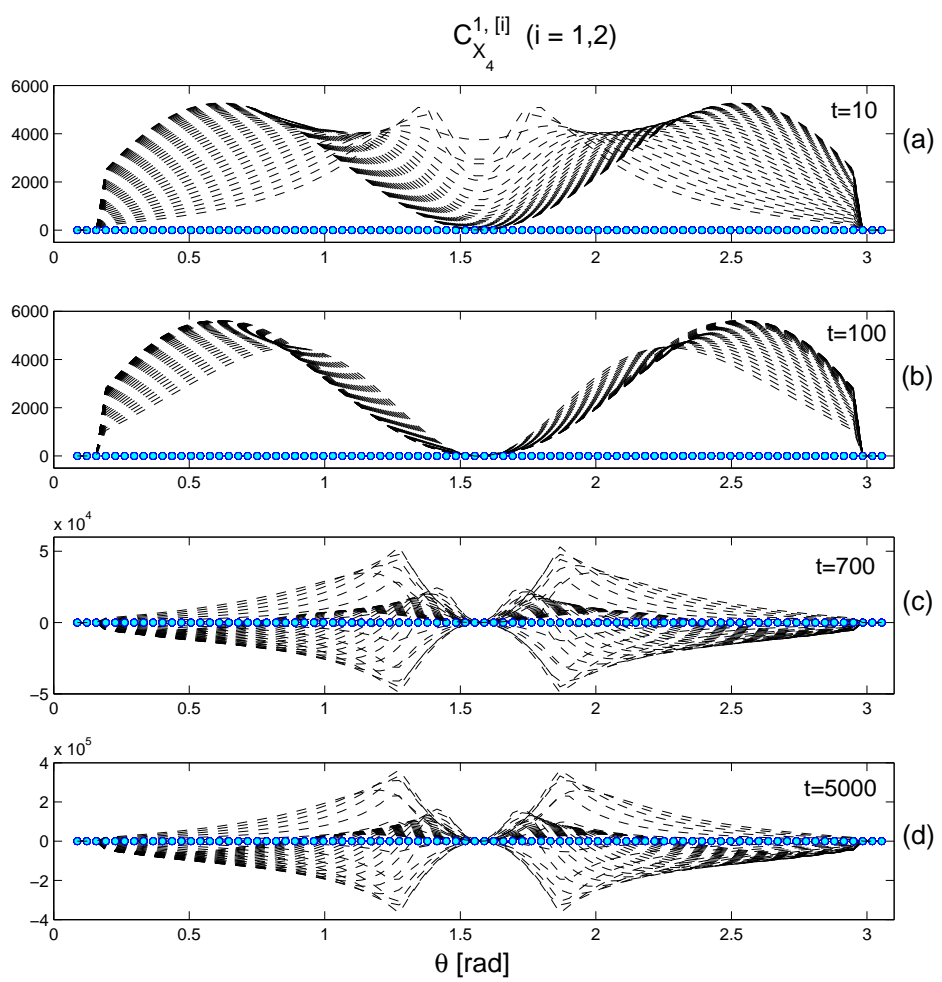

Figure 4. Asymptotic divergence of the densities $C_{X_{4}}^{1,[2]}$ (black dashed lines) and $C_{X_{4}}^{1,[1]}$ (blue circles) shown as a two-dimensional plot versus $\theta$ for several values of $\phi \in(0,2 \pi)$. 


\section{Conclusion}

The present study is devoted to calculation of conserved vectors and understanding their asymptotics for the nonlinear model describing nonlinear nonstationary flows on a rotating spherical surface. The model involves finite disturbances that are superimposed to a zonally averaged mean flow having only a latitude dependence. In terms of atmospheric modeling, the exact solutions discussed here are used to describe physically relevant zonal flows. As has been discussed in [11] and [4], under the assumption of no friction, and a distribution of temperature dependent only upon latitude and altitude, the presence of the Coriolis force creates a cyclonic rotation around the poles, i.e., west-to-east winds. The special attention in this letter was given to analyzing and visualizing the conserved densities $C^{1}$ associated with some of the known symmetries. Particularly, it has been illustrated that the asymptotic behavior of the conserved densities can differ greatly depending on the selection of symmetry and the choice of the invariant solution. However, the only choice for the function $F(\theta)$ has been made in our work. Additionally, for the purpose of illustration, the singularities associated with the invariant solutions were removed artificially by truncating the spherical layer at the two rings located in the northern and southern hemispheres so that the velocity components and pressure terms associated with the invariant solutions are free of pole singularities.

From mathematical standpoint, the velocity and the pressure terms are unbounded in the neighborhood of the pole. Unbounded terms are common in equations posed in spherical coordinates and it introduces a host of computational problems that are collectively known as the "pole problem" (see e.g., [31] for the nomenclature). The treatment of the geometric singularity in spherical coordinates has for many years been a difficulty in the development of analytic and numerical simulations for oceanic and atmospheric flows around the Earth ([5],[6], [7],[9]). The common results on computational experiments (see e.g., [5]; [23]; [35] and [32]) seem to provide credible evidence to support the assertion that singular solutions to the shallow water equations may exist on a stationary sphere. They also suggest that singular solutions are less likely on a rotating sphere. However, the experiments conduced up to the date are not sufficiently extensive to support any credible assertions about the existence or nonexistence of singular solutions to the shallow water equations on a rotating sphere. Even less is known about singular terms used on a spherical surface. The results of the forthcoming studies will appear elsewhere.

Acknowledgements. We acknowledge the financial support of the Government of Russian Federation through Resolution No. 220, Agreement No. 11.G34.31.0042.

\section{References}

[1] R.F Anderson, S. Ali, L.L. Brandtmiller, S.H.H. Nielsen, M.Q. Fleisher. Wind-driven upwelling in the Southern Ocean and the deglacial rise in atmospheric $\mathrm{CO}_{2}$. Science. (2006) 323, 1443-1448.

[2] G.K. Bachelor. An Introduction to Fluid Dynamics. Cambridge University Press, (1967) Cambridge.

[3] S. Balasuriya. Vanishing viscosity in the barotropic $\beta$-plane J. Math.Anal. Appl., (1997) 214, 128-150.

[4] O.M. Belotserkovskii, I.V. Mingalev, O.V. Mingalev. Formation of large-scale vortices in shear flows of the lower atmosphere of the earth in the region of tropical latitudes. Cosmic Research, (2009) 47, (6), 466-479.

[5] G. Ben-Yu. Spectral method for vorticity equations on spherical surface. Math. Comput. (1995) 64, $1067-1079$.

[6] E.N. Blinova. A hydrodynamical theory of pressure and temperature waves and of centres of atmospheric action. C.R. (Doklady) Acad. Sci USSR, (1943) 39, 257-260.

[7] E.N. Blinova. A method of solution of the nonlinear problem of atmospheric motions on a planetary scale. Dokl. Acad. Nauk USSR, (1956) 110, 975-977.

[8] C. Cenedese, P.F. Linden. Cyclone and anticyclone formation in a rotating stratified fluid over a sloping bottom. J. Fluid Mech., (1999) 381, 199-223.

[9] A. Furnier, H. Bunger, R. Hollerbach, I. Vilotte. Application of the spectral-element method to the axisymetric NavierStokes equations. Geophys. J. Int. 156, (2004) 682-700.

[10] H. Golovkin. Vanishing viscosity in Cauchy's problem for hydromechanics equation. Proc. Steklov Inst. Math. (1966) 92, 33-53.

[11] E. Herrmann. The motions of the atmosphere and especially its waves. Bull. Amer. Math. Soc. 2 (9), $285-296$.

[12] P.A. Hsieh. Application of modflow for oil reservoir simulation during the Deepwater Horizon crisis. Ground Water. (2011) 49 (3), 319-323. 
[13] R.N. Ibragimov. Nonlinear viscous fluid patterns in a thin rotating spherical domain and applications. Phys. Fluids. (2011) 23, 123102.

[14] R.N. Ibragimov, M. Dameron. Spinning phenomena and energetics of spherically pulsating patterns in stratified fluids. Physica Scripta. (2011) 84, 015402.

[15] N.H. Ibragimov, R.N. Ibragimov. Intergarion by quadratures of the nonlinear Euler equations modeling atmoaspheric flows in a thin rotating spherical shell. Phys. Letters A. (2011) 375, 3858.

[16] N.H. Ibragimov, R.N. Ibragimov. Applications of Lie Group Analysis in Geophysical Fluid Dynamics. (2011) Series on Complexity, Nonlinearity and Chaos, Vol 2, World Scientific Publishers, ISBN: 978-981-4340-46-5.

[17] N.H. Ibragimov, R.N. Ibragimov. Conservation laws and invariant solutions for a model of nonlinear atmospheric zonal flows in a thin rotatimng spherical shell. (2012) Archives of ALGA, vol. 9, pp.27-38.

[18] R.N. Ibragimov, D.E. Pelinovsky. Effects of rotation on stability of viscous stationary flows on a spherical surface. Phys. Fluids. (2010) 22, 126602.

[19] R.N. Ibragimov. Mechanism of energy transfers to smaller scales within the rotational internal wave field. Springer. Mathematical Physics, Analysis and Geometry, (2010) 13 (4), 331-355.

[20] R.N. Ibragimov, D.E. Pelinovsky. Incompressible viscous fluid flows in a thin spherical shell. J. Math. Fluid. Mech. (2009) 11, 60-90.

[21] R.N. Ibragimov. Shallow water theory and solutions of the free boundary problem on the atmospheric motion around the Earth. Physica Scripta. (2000) 61, 391-395.

[22] N.H. Ibragimov. A new conservation theorem. Journal of Mathematical Analysis and Applications, (2007) 333 (1), $311-328$.

[23] D. Iftimie, G. Raugel. Some results on the Navier-Stokes equations in thin 3D domains. J. Diff. Eqs. (2001) 169, 281-331.

[24] H. Lamb. Hydrodynamics. Cambridge University Press, 5th edition (1924) .

[25] J.L. Lions, R. Teman, S. Wang. On the equations of the large-scale ocean. Nonlinearity. (1992) 5, $1007-1053$.

[26] J.L. Lions, R. Teman, S. Wang. New formulations of the primitive equations of atmosphere and applications. Nonlinearity, (1992) 5, 237-288.

[27] E. Noether. Invariante Variationsprobleme. Konigliche Gessellschaft der Wissenschaften, Gottingen Math. Phys. K1., (1918) English transl.: Transport Theory and Statistical Physics 1(3) (1971) 186-207.

[28] D.T. Shindell, G.A. Schmidt. Southern Hemisphere climate response to ozone changes and greenhouse gas increases. Res. Lett., (2004) 31, L18209.

[29] J. Shen. On pressure stabilization method and projection method for unsteady Navier-Stokes equations, in: Advances in Computer Methods for Partial Differential Equations, (1992) 658-662, IMACS, New Brunswick, NJ.

[30] C.P. Summerhayes, S.A. Thorpe. Oceanography, An Illustrative Guide. (1996) New York: John Willey \& Sons.

[31] P.N. Swarztrauber. Shallow water flow on the sphere. Mon. Wea. Rev. (2004) 132, 3010-3018.

[32] P.N. Swarztrauber. The approximation of vector functions and their derivatives on the sphere. SIAM J. Numer. Anal. (1981) 18, 181-210.

[33] R. Temam, M. Ziane. Navier-Stokes equations in thin spherical domains. Contemp. Math. (1997) 209, $281-314$.

[34] J.R. Toggweiler, J.L. Russel. Ocean circulation on a warming climate. Nature. (2008) 451, $286-288$.

[35] W. Weijer, F. Vivier, S.T. Gille, H. Dijkstra. Multiple oscillatory modes of the Argentine Basin. Part II: The spectral origin of basin modes. J. Phys. Oceanogr., (2007) 37, 2869-2881.

[36] D. Williamson. A standard test for numerical approximation to the shallow water equations in spherical geometry. J. Comput. Physics., (1992) 102, 211-224. 